\title{
Orts- und Namenregister
}

Aachen 240

Abbo v. Metz 457f.

Adalgudis 316-318

Aegidius 60, 64, 68f.

Aeneas 336, 338-340, 344, 358, 362, 365, 366 A., 369, 371-373

Aeonius v. Arles 447 A.

Aetherius v. Lyon 132 A.

Aëtius, Fl. 25, 35-41, 63, 65, 261f., 361f., 455

Afrika 28, 35, 40, 45-47, 52-54, 65, 67, 84-86, 102 A., 103, 159, 288, 357 A., 362-364, 366-368, 370, 436 A.

Agaune (Saint-Maurice en Valais) 379f., 389

Agde 117, 118 A.

Agen (Aginnum) 160 A., 165 A.

Agroecius v. Sens 390f.

Ägypten 102 A.

Aigatheus 316-318

Aix-en-Provence 103 A., 202f., 205 (fig. 2), 206, 212 (fig. 7), 213, 227

Alarich I. 25, 27, 30, 82f., 87, 368 A., $371 \mathrm{~A}$

Alarich II. 88

Albi 456-460, 462f.

Alcima, Tochter des Sidonius Apollinaris 464

Alcimus Ecdicius Avitus s. Avitus v. Vienne

Alcuin 415

Alexander v. Aphrodisias 343

Alpes Maritimae 211, 218

Altaripa 463

Amantius, Märtyrer 457

Ambrosius Aurelius 455

Ambrosius v. Mailand 131 A., 422 A., 427 A., 440-443

Amida (Diyabarkir) 287

Amiens (Samarobriva) 158 A., 160 A., 226 A.

Anaolsus 36

Andelot 223-225, 233, 239, 243

Andemantunum $s$. Langres
Anianus, vir spectabilis 88

Anianus v. Orléans 132 A.

Antalas 288

Anthemius, F1. Procopius, röm. Kaiser 6265 A., 69, 356f., 371-373

Antibes 209

Antonius, M. 393

Apollinaris, Großvater des Sidonius Apollinaris 26

Apollinaris, Sohn des Sidonius Apollinaris 61, 427 A., 428 A.

Apollinaris Sidonius $s$. Sidonius Apollinaris, C. Sollius

Apollinaris v. Valence 114 A.

Aprunculus v. Langres 184 A.

Apt 203

Aquae $s$. Dax

Aquae Sextiae 202

Aquitanien 27f., 33-35, 50, 66, 79, 86, 97, 105, 153f., 156 A., 158 A., 162 A., 176, 177 A., 218, 238, 266, 269, 429 A., 446 A.

Aquitania I 153f., 170 A.

Aquitania II 27, 34, 153f., 169 A.

Arator 409, 410

Arbogast v. Chartres 59, 402f., 407

Arborius 61

Arelate $s$. Arles

Arelatensis provincia 218

Argelliers 218

Aristoteles 343, 442 A.

Arles (Arelate) 27, 34, 36, 62, 67, 103 A., 107 A., 108 A., 109, 136 A., 153, 154 A., 202f., 205f., 209, 210 (fig. 5), 213, 218, 226f., 229 A., 413, 428 A., 436 A., 447 A.

Armorica 255 A., 258, 261f., 270

Arnulf v. Metz 456f.

Arpinum 423

Arvandus 64, 87

Arvernia 357, 370

Athaulf, König der Westgoten 25-29, 31, 64, 83 
Attalus, Priscus, röm. Kaiser 27-29, 34, 69

Attia Viriola 425f.

Auch 165 A.

Audoenus/Dado v. Rouen 115 A., 456458,462

Augsburg 55

Augst (Colonia Augusta Raurica) 161 A.

Augustinus v. Hippo 72, 103 A., 120, 129 A., 337, 405f., 409 A., 415, 427 A., 429 A, $440 f$.

Augustodunum $s$. Autun

Augustoritum $s$. Limoges

Augustus, röm. Kaiser 69, 153, 158 A., 207

Aulus Cluentius 425

Aurelianus, röm. Kaiser 184

Ausonius, Decimus Magnus 69-71, 73, 176f., 185-188, 331-349, 399f. A.

Austrasien 237f.

Autun (Augustodunum) 168 A.

Auvergne 61, 65

Auxerre 103f. A., 242

Avignon 209

Avita, Schwester des Desiderius of Cahors 464

Avitus, Fl. Eparchius, röm. Kaiser 38-40 $60,62,64,66,69,86,356-358$, 363 A., 368, 370, 373 A., 401, 424 A.,

Avitus v. Clermont 461

Avitus v. Vienne 60f., 65, 87, 424 A., 463f.

Bacaudae/Bagaudae 59, 73, 255-259, 261-263, 266-273

Baetica 30

Bagacum $s$. Bavay

Barcelona 40, 105

Bavay (Bagacum) 159f. A.

Bazas 28

Beda Venerabilis 411 A., 416

Belgica 155 A., 160 A., 162 A.

Belgica I $155 \mathrm{~A}$.

Belgica II 155 A., 163 A., 164 A., 286

Belisarius, F1. 455

Berimund 37

Bero 322

Bertegyselus 462

Bliesbruck $161 \mathrm{~A}$.

Blithildis, Großmutter des Arnulf v. Metz $466 \mathrm{~A}$.

Boethius, Anicius Manlius Severinus 307 A., 455

Bonifatius 84
Bonitus v. Clermont 461

Bordeaux (Burdigala) 28, 70, 159 A., 165 A., 170 A., $172-177,181$ A., 187f., 202, 207, 331, 399 A., 400 A.

Bourges 91f., 100, 164f. A., 215, 318 A.

Bretagne 60

Britannien 429 A.

Brunhild 223f.

Burdigala $s$. Bordeaux

Burgund 231, 237f., 240

Burgundofaro v. Meaux 457

Burgus 61

Cadolaicus 321

Caelestinus v. Rom 109

Caesar, C. Iulius 153, 399

Caesarius v. Arles 107 A., 114 A., 117, 135f. A., 205, 413-415, 428 A., $436 \mathrm{~A}$.

Caesarodunum $s$. Tours

Cahors 457f., 464

Calminius 61

Candidus, Märtyrer der thebäischen Legion 380

Caper, Fl. 390-392

Capo Don 218

Carausius, M. Aurelius, röm. Kaiser 68

Carcassonne 209

Cassianus, Johannes 71

Cassiciacum $120 \mathrm{~A}$.

Cassiodorus, Fl. Magnus Aurelius 46f., 414

Cassius, C. 435

Castinus 84

Castrum Rauracense $s$. Kaiseraugst

Catilina 393

Cato d.Ä. 392

Cato d.J. 392

Catullinus 280

Censorius v. Auxerre 433

Ceraunia 431f., $435 \mathrm{f}$.

Chalkedon $108 \mathrm{~A}$.

Charibert I. 223f.

Charibert II. 223, 238

Châteaudun $231 \mathrm{~A}$.

Châteauneuf-de-Grasse 218

Childebert II. 223f., 238

Childebert III. 318 A., 322-324

Childerich I. 282, 284-287

Childerich II. 238 A., 323

Chilperich I. 118, 223, 239

Chlodocharius (i.e. Chlodwig III. oder Chlothar IV.) 323

Chlodwig I. 53, 103 A.

Chlodwig II. 323 
Chlodwig III. s. Chlodocharius

Chlotharius (i.e. Chlothar I. oder Chlothar II.) 223, $466 \mathrm{~A}$.

Chlothar II. 224, 235f., 238, 240, 456, 458

Chlothar IV. s. Chlodocharius

Chrocus, König der Alamannen 68, 162 A., 163 A., 174 A.

Cicero, M. Tullius 384f, 387, 390-393, 408, 409 A., 415, 416 A., 423, 425f., 432, 435-437, 440-442, 444f., 447

Cicero, Qu. Tullius 392f.

Cimiez 206

Cimitile 105, 106 A., 108, 111 A.

Claudianus Mamertus 411

Claudianus, Cl. 284f., 368 A., 370 A., 371

Clermont (Augustonemetum/Arverni) 60, 62, 65, 67, 162f. A., 164f. A., 166, 167 (Abb. 2), 174 A., 183, 184 A., 188, 203, 215, 216 (fig. 10), 229, 230 A., 232 A., 242, 464

Clichy 240

Clos-de-la-Lombarde 215

Col de Panissars 32

Colonia Agrippinensis $s$. Köln

Colonia Augusta Raurica $s$. Augst

Compiègne $319 \mathrm{~A}$.

Consentius, Schwiegervater des Iovinus 26

Consentius, Sohn des Consentius 186

Consorannorum civitas s. Saint-Girons und Saint-Lizier

Constantinus I., röm. Kaiser 70f., 73, 226, $400 \mathrm{~A}$.

Constantinus (III.), Fl. Claudius, röm. Kaiser 26, 31f., 60, 64, 68, 83, 103 A.

Constantius Chlorus, röm. Kaiser 403

Constantius III., röm. Kaiser 27-32, 34f., $50,65,83 \mathrm{f}$.

Constantius v. Lyon 110 A., 257, 261f., 413, 421-424, 426, 440

Constantius v. Albi 462f.

Cornelius Cethegus 455

Convenarum civitas $s$. Saint-Bertrand-deComminges

Cornificius, Qu. 435

Cularo $s$. Grenoble

Cyprian v. Karthago 427 A., 444

Dagobert I. 74, 240, 456-458, 462

Dalheim (vicus Ricciacum) 162 A.

Dalfinus v. Saint-Denis 321-323

Damasus v. Rom 332

Dardanus, Cl. Postumus 27, 61

Dax (Aquae) 165f. A.

Decaniacum 464
Decimus Rusticus 26

Defensor v. Ligugé 459

Demosthenes 408

Desiderius v. Cahors 74, 135 A., 239, 241, 429 A., 449, 455-467

Desiderius v. Vienne 411, 416 A.

Desiderius v. Albi 456

Dido v. Albi 459

Die 202, 209

Dijon (Divio) 183-185, 186 A.

Diocletianus, röm. Kaiser 164, 226, 307, 399 A.

Domitianus v. Angers 132 A.

Domnicius 282

Donatus, Aelius 412, 415

Divio $s$. Dijon

Draguignan 218

Drogo, Sohn Pippins II. 306 A., 312 A.

Dynamius patricius 115 A., 132 A., 224, 239

Éauze 165 A., 203, 209

Ebroin 242, 322

Eligius v. Noyon 115 A., 121 A., 242, 456f., 459, 462

Ennodius, Magnus Felix 130 A., 424 A.

Entremont 206

Eparchius, Sohn des Ruricius v. Limoges $427 \mathrm{~A}$

Ermelinus 313 A.

Eucherius v. Lyon 71, 107 A., 379-394, 424 A., $439 f$.

Eudoxius, Anführer der Bagaudae 262

Eufronius v. Tours 131 A., 134 A.

Eumenius, gallischer Panegyriker 403

Euplutius 30

Eurich, König der Westgoten 66, 87, $429 \mathrm{~A}$.

Eusebius v. Vercelli 103 A.

Eutropius v. Orange 114 A., 133, 134 A.

Exsuperius v. Toulouse $131 \mathrm{~A}$.

Exsuperius, Märtyrer der thebäischen Legion 380

Faesulae 448 A.

Faustus v. Riez 108 A., 427 A., 429, 436 A., 461

Felix, Fl. 84

Felix v. Nola 104f., 106 A.

Felix v. Limoges 465

Ferreolus, Tonantius, Prätorianerpräfekt v. Gallien 405, 411, 466 A.

Firminus v. Uzès $466 \mathrm{~A}$.

Florentius $441 \mathrm{~A}$.

Francia 230f., 235, 238 A., 240 
Fredegar 238, 240, 459

Friedrich, Sohn Theoderichs I. 84, 86f.

Fréjus 212 (fig. 7), 213

Fronto, M. Cornelius 423, 425, 426 A.

Fundi 332

Gaius, römischer Jurist 304-306, 307 A.

Galla Placidia 27f., 30f., 36, 38, 84

Gallaecia 83

Galliarum provinciae $36,39,79,153,157$, $159,225,267,270$

s. auch Alpes Maritimae, Aquitania, Belgica, Narbonensis, Novempopulana Viennensis

Gallien 25-29, 41, 45, 48, 50f., 53f., 5967, 71f., 74f., 80f., 83, 85f., 88, 92 94, 96f., 99-102, 103 A., 106 A., 107-110, 116-123, 125, 127 A., 129 A., 130, 132, 135f., 156f., 158 A., 159 A., 161 A., 162-164, 166 A., 168 A., 174-176, 181, 185, 187, 190f., 201, 212, 215, 218, 225234, 243, 255, 256 A., 257 A., 258, 261f., 269, 273, 277-279, 286-288, 307, 331-349, 357 A., 358, 363 A., 364 A., 394, 399-401, 403f., 410, 412-414, 416, 424 A., 430, 438, 440, 444f., 446 A., 447 A., 449, 458-460, 464, 466f.

Gallienus, röm. Kaiser 162 A.

Galswinth 223f.

Gammo 316-318

Gap 202, 209

Garonne 66

Geiserich, König der Vandalen 40, 52, 357f., 361-364, 368

Genf (Genava) 178f. mit Abb. 8, 180 A., 202, 209, 211 (fig. 6), 212

Gennadius v. Marseille 414

Genovefa 111 A., 117 A.

Germanicus 99 A., 283

Germanus v. Auxerre 103f. A., 107 A., 109, 110 A., 111f., 132 A., 261f., 413, $421 \mathrm{~A}$.

Germanus v. Paris 132 A.

Gerontius 26

Gildo 371

Gimbercthus 322

Glycerius, röm. Kaiser 86f.

Goar, König der Alanen 26

Gordianus III., röm. Kaiser 159 A.

Gratianus, röm. Kaiser 331

Gregor v. Langres 114, 184
Gregor v. Tours 68 A., 74f., 93 A., 95 A., 97, 98 A., 99 A., 115, 129 A., 131 A., 136 A., 154 A., 157 A., 163 A., 174 A., 183-185, 186 A., 188f., 207, $209,215,218,223-225,227,229$, 232-238, 242, 401, 404, 410, 411 A., 460

Gregor der Große 411f., 413-416, 455

Grenoble (Cularo) 164, 202, 209, 214

Grimoald 312f., 315, 320-323

Gundahar, König der Burgunder 26

Gundioch, König der Burgunder 86

Gundobad, König der Burgunder 86

Guntram I., König der Franken 223f.

Guntram Boso 224

Hagenbach 162 A.

Heraclianus 28

Heros v. Arles 103 A.

Hesperius 427 A., 428 A.

Hiberia, Gattin des Ruricius v. Limoges $424 \mathrm{~A}$.

Hieronymus 105 A., 121, 408, 414, 440, $441 \mathrm{~A}$.

Hilarius v. Poitiers 427 A.

Hilarius v. Arles 103f. A., 107 A., 109112, 114 A., 128f. A., 131 A.

Hippo 103 A.

Hispanien $s$. Spanien

Honoratus v. Arles 107 A., 109, 131 A., 135 A., 235 A.

Honorius, röm. Kaiser 26-28, 30f., 66, 84, 371 A., 401

Horatius Flaccus, Qu. 405-407, 409 A., $415,428 \mathrm{~A}$.

Hydatius 26, 30, 33, 36-39, 256 A., 257

Ildefons v. Toledo 414

Illyricum 102 A.

Iovinus, röm. Kaiser 25-28, 32, 64

Isidor v. Sevilla 416

Italien 25, 27f., 35, 45-48, 50f., 53f., 64f., 67f., 79f., 85-87, 102 A., 103, 154 A., 201, 231 A., 240 A., 261f., 300 A., 349, 357, 365f., 368 A., 370, 371,401

s. auch Ligurien, Kampanien

Iulius Nepos, röm. Kaiser 65

Iulius Titianus 423

Johannes, grammaticus 74

Jordanes 26, 31-33, 37, 39f.

Justinian I., röm. Kaiser 65, 399, 455 
Kaiseraugst (Castrum Rauracense) 161 A. Kampanien 105

Karl der Große 240, 415

Karthago 28, 40, 360-362, 365-372

Köln (Colonia Agrippinensis) 156 A., 159 A., 439

Konstantinopel 26, 32f., 40, 66, 73

Langres (Andemantunum/Lingonica urbs) 114

Latiniacus $321 \mathrm{f}$.

Lazarus v. Aix 103 A.

Leander v. Sevilla 412

Le Mans 170 A., 171 A., 242

Leo, consiliarius der Westgotenkönige 87

Leo I., röm Kaiser 357, 372

Lérins 103, 107, 108 A., 109, 112f., 120f., 413, 424 A., 429 A., 439 A.

Leudegarius v. Autun 459

Ligurien 218

Limoges (Augustoritum/urbs Lemovica) 156 A., 158, 182, 428 A., 464

Limonum $s$. Poitiers

Litorius 37-39, 85, 261

Livia, Gattin des Augustus 207

Livius, Titus $409 \mathrm{~A}$.

Loire 83 A., 85, 88, 97 A., 99, 104 A., 201

Lugdunum $s$. Lyon

Lugdunum Convenarum $s$. Saint-Bertrandde-Comminges

Lucanus, M. Annaeus 337 A.

Lupus v. Troyes 61, 107 A., 109, 110 A.

Lupus, dux 224

Lupus v. Ferrières $416 \mathrm{~A}$.

Lusitania 30

Luzarches 319 A.

Lyon 62, 66f., 71, 79, 132 A., 214, 363, $421 \mathrm{~A}$.

Mâcon 118 A.

Macrobius, Ambrosius Theodosius 339, $421 \mathrm{~A}$.

Magnentius, Fl. Magnus, röm. Kaiser 32, 68

Magnus Maximus, röm. Kaiser 68, 129 A., 401

Mainz 242

Maiorianus, Fl. Iulius Valerius, röm. Kaiser 62-64, 69, 86f., 356f., 363f., 366-369

Mamacas $s$. Montmacq

Mamertinus, lateinischer Panegyriker 255, 257

Mamertus v. Vienne 110f.

Marcella 408
Marmoutier 103 A., 104, 106, 108, 111

Maroveus v. Poiters 236 A.

Marseille 27, 212f., 214f., 239

Martialis v. Limoges $182 \mathrm{~A}$.

Martin v. Tours 100-112, 120 A., 121 A., 129 A., 131 A., 134 A., 236, 322, 413,416

Massiliensis provincia 218

Masuna, rex gentium Maurorum et Romanorum 288

Mauritius, Märtyrer der thebäischen Legion $379 f$.

Maximianus, röm. Kaiser 164, 255, 380, 389

Maximus, Sohn des Gerontius und röm. Kaiser 26

Maximus v. Lérins 108 A.

Maximus v. Riez 115 A., 132 A., 209

Mediolanum $s$. Saintes

Melania d. Ä. 102 A., 121

Merobaudes, F1. 255 A., 262, $361 f$.

Merowech II. 118

Metz 164 A., 229 A., 242

Minucius Felix 441

Montmacq (Mamacas) 319 A., 321-324

Namatius, Feldherr im Dienst der Westgoten 61

Namatius, Schwager des Ruricius v. Limoges (möglicherweise identisch mit dem Vorigen) 431f., 434-436

Narbo $s$. Narbonne

Narbonensis 26, 32, 109 A., 153 A., 202, 209

Narbonensis I 209

Narbonensis II 202, 209

Narbonne (Narbo) 27f., 37-39, 62, 185187, 201, 209

Neustrien 237f.

Nicetas v. Remesiana 102 A.

Nicetius v. Lyon 114, 132 A.

Nicetius v. Trier 130 A.

Nicomachus Flavianus 449 A.

Nîmes 203, 207, 209

Nola 102 A., 104f., 443 A.

Notre-Dame-du-Brusc 218

Novempopulana 153, 154 A., 155 A., 165, 166 A., 203, 207, 208 (fig. 4), 211

Odoaker, König von Italien 45, 53, 54 A., 69

Olybrius, Fl. Anicius, röm. Kaiser 86f.

Olympiodoros v. Theben 27f., 30

Ommatius, Sohn des Ruricius v. Limoges 427 A., 429 
Oppedette 218

Orestes 47, 54

Orosius, Paulus 26, 30, 33

Ovidius Naso, P. 335, 341, 345

Palatin 359

Panaitios 432 A.

Papianilla, Gattin des Sidonius Apollinaris $424 \mathrm{~A}$.

Paris 60, 66, 111 A., 164 A., 174 A., 178 A., 180 A., 240, 242, $323 f$.

Pascentius v. Poitiers 132 A.

Paulinus v. Nola 102, 104-107, 120f., 331f., 349, 440, 443 A., 448 A.

Paulinus v. Périgueux 413 A.

Paulus, Apostel 105 A., 332, 436 A., 444

Paulus, comes 59, 71

Paulus v. Verdun 456f., 462, 465

Pausanias 334

Pelagia, Gattin des Aetius 40

Périgueux 165 A., 169, 178 A., 207

Petrus, Apostel 105 A., 332

Petronius, Fl. Maximus, röm. Kaiser 357

Placidina, Schwiegertochter des Sidonius Apollinaris 464

Platon 384, 392

Plinius d. J., C. Caecilius Secundus 348, 387, 421-423, 425, 426 A., 428 A., 443 A., 445

Poitiers (Limonum) 156 A., 165 A., 166, 178 A., 181 A., 215, 232 A., 234 A.

Pomerius, Iulianus 413, 415, 436

Pontius Leontius 61

Postumus, röm. Kaiser 62

Pouan 284

Primuliacum 104f., 106 A., 108, 111 A., $331,332 \mathrm{~A}$.

Priscillianus v. Avila 105

Priscus v. Lyon 132 A.

Proculus Gregorius 332

Prokopios v. Caesarea 47 A., 54

Prosper Tiro 30, 34, 36-38, 72, 85, 401 A.

Prosper v. Orléans 132 A.

Provence 97, 218, 227, 231, 233 A., 439

Prudentius, Aurelius Clemens 405-407. $409 \mathrm{~A}$.

Pyrenäen 26, 32f., 35

Radagaisus 82

Ravenna 305 A., 307 A.

Reims 66, 164 A., 172 A., 176 A., 178 A., 180 A., 182 A., 183 A.

Rhone, Rhoneraum 97, 107, 120 A., 202, 203

Ricimer 65, 86
Riez 209

Rigofredus, 314 A., 321-223

Riothamus 59

Rodez 170 A.

Rom 66, 70-72, 102 A., 105 A., 187 A., 357f., 360-366, 368-372, 409

Romulus Augustus 455

Roujan 218

Rufinus v. Aquleia 121, 405, 407

Ruricius v. Limoges 230, 422 A., 424 $440,443-449,461,463-465$

Rusticus v. Narbonne 187, 460

Rusticus v. Cahors, Bruder des Desiderius of Cahors 456f., 462

Rutilius Namatianus, Cl. 59, 64, 69, 255 $257 \mathrm{~A}$.

Sabinus v. Piacenza 441

Sagittarius v. Gap 98 A.

Saint-Bertrand-de-Comminges (Lugdunum Convernarum/civitas Convenarum) 35, 155 A., 165 A., 177 A., 203, 207f., 213

Saint-Césaire (Arles) 213

Saint-Denis 239 A., 310f., 313-315, 319322

Saintes (Mediolanum) 158 A., 160 A., 165 A., 169 A., 170 A., 171 (Abb. 4), 202, 207

Saint-Germain-des-Prés 316-318

Saint-Girons 165 A.

Saint-Hermentaire (bei Draguignan) 218

Saint-Just (Lyon) 214

Saint-Laurent (Grenoble) 214

Saint-Lizier 35, 165 A.

Saint-Maurice en Valais $s$. Agaune

Saint-Maximin (bei Draguignan) 218

Saint-Victor (Marseille) 214

Sallustius, C. Crispus 409 A.

Salonius v. Genf 107 A.

Salonius v. Embrun 98 A.

Salvian v. Marseille 39, 72f., 255-273, 439f., 443, 445

Salvius v. Albi 456

Samobriva $s$. Amiens

Sapaudia 41

Sarus 83

Saturninus v. Toulouse 207

Scipio Africanus, P. Cornelius 393

Sebastianus, röm. Kaiser 27, 37, 39f.

Seneca, Lucius Annaeus 381 A., 384

Septicius, C. Clarus 422

Septimania 231

Seronatus 65

Servius 339 
Severus, Fl. Libius, röm. Kaiser 87

Sidonius Apollinaris, C. Sollius 38-40, 60-63, 65f., 69, 73, 91f., 99 A., 107f., 108 A., 110 A., 111 A., 113, 118 A., 132 A. ,186f., 229f., 255 A., 262 A., 280-284, 288, 355-373, 401-415, 421-431, 433 A., 435 A., 438, 440, 444f., 448f., 461, 463f.

Sigerich, König der Westgoten 83

Sigibert I. 223, 231 A.

Sigibert III. 456f.

Sigismer 282-285, 287

Silius Italicus 337 A., 348, 368 A.

Simplicius v. Bourges 91

Sisteron 202

Soissons 60, 242

Spanien 25f., 29-35, 38, 40, 53, 80, 83, 105f., 159 A., 234 A., 255, 256 A., 258, 267, 349, 458

s. auch Baetica, Lusitania, Tarraconensis

Statius, P. Papinius 335, 337 A., 428 A.

Stilicho, Fl. 83, 284f., 448 A., 455

Sulpicius Severus 102, 104-107, 111 A., 120f., 129 A., 331f., 400, 410, 413, $441 \mathrm{~A}$.

Sulpicius I. v. Bourges 460

Sulpicius II. v. Bourges 457, 460, 462f., 465

Syagria, Mutter des Bonitus of Clermont 461

Syagrius, Sohn des Aegidius 59f., 68f., 455

Syagrius patricius, Bruder des Desiderius v. Cahors 456f., 462

Symmachus, Qu. Aurelius 421-423

Tacitus 381 A., 383 A.

Tarraconnensis 33

Theoderich I., König der Westgoten 36$40,66,83,85$

Theoderich II., König der Westgoten 66, 86,357

Theoderich d. Gr., König der Ostgoten 45, 53

Theodor v. Fréjus 108 A.

Theodor v. Marseille 239

Theodosius I., röm. Kaiser 31, 33, 209, 401

Theophrast 432 A.

Theopolis 61

Theuderich III. 323

Tibatto, Anführer der Bagaudae 261f., 269

Timotheus, comes unter den Westgoten 88
Toulouse (Tolosa) 33f., 37, 39f., 86, 114 A., 131 A., 153, 154 A., 168 A., 203, 204 (fig. 1), 207, 213, 229 A.

Tour de Vésone 207

Tournai (Turnacum) 163 A., 284

Tourraine 97

Tours (Caesarodunum) 103f., 111, 115 A., 131 A., 160 A., 162 A., 164 A., 172 A., 176 A., 178, 182, 226 A., 227, 228 A., 229 A., 234f

Traianus, M. Ulpius, röm. Kaiser 358, $387,426 \mathrm{~A}$.

Trier 62f., 66, 79, 153, 154 A., 226, 227 A., 331f., 333 A., 399 A., 402f., 439

Trinquetaille 202

Turnacum $s$. Tournai

Uernus 321f.

Urbicus von Riez 132 A.

Ursicinus 439

Vadomarius, König der Alamannen 83

Valenciennes 319 A.

Valentine 177

Valentinian I., röm. Kaiser 285

Valentinian III., röm. Kaiser 38, 84, 261, 357

Valerianus, röm. Kaiser 162 A.

Valerianus v. Cimiez 386

Valerius Flaccus, C. 334

Varro, M. Terentius 405-407, 409 A.

Venantius Fortunatus, Honorius Clementianus 114 A., 115, 130 A., 131 A., 132 A., 134 A., 135 A., 403, 413 A.

Venerandus 463

Veranus v. Vence 107 A.

Vercelli 408 A.

Verecundus, grammaticus 120 A.

Vergilius, P. Maro 69, 281, 333, 335, 336342, 345f., 362, 365 A., 407, 409 A., $415 f$., 466

Verus v. Rodez 462

Verus v. Orange 114 A., 133

Vetranio, röm. Kaiser 68

Victor v. Vita 47 A., $51 \mathrm{f}$.

Victorius 61

Vienne 66, 207, 209

Viennensis 109 A.

Vincentius 61

Vitericus 37, 40

Vivianus v. Saintes 114 A.

Vuistrilingo 457 
Wallia, König der Westgoten 30, 32, 83

Wandregisel, Klostergründer von Fontenelle 457
Zenon, röm. Kaiser 69

Zosimos 59, 255 A., 256 A 